\title{
Predictive Factors for Future Onset of Reflux Esophagitis: A Longitudinal Case-control Study Using Health Checkup Records
}

\begin{abstract}
Yuzuru Toki, ${ }^{1}$ Ryo Yamauchi, ${ }^{2}$ Eizo Kayashima, ${ }^{3}$ Kyoichi Adachi, ${ }^{4}$ Kiyohiko Kishi, ${ }^{5}$ Hiroshi Suetsugu, ${ }^{6}$ Tsuneya Wada, ${ }^{7}$ Hiroyoshi Endo, ${ }^{8}$ Hajime Yamada, ${ }^{9}$ Satoshi Osaga, ${ }^{10}$ Takeshi Kamiya, ${ }^{10 *}$ Koji Nakada, ${ }^{11}$ Katsuhiko Iwakiri, ${ }^{12}$ Ken Haruma, ${ }^{13}$ and Takashi Joh ${ }^{14}$

${ }^{\prime}$ Department of Internal Medicine, Hidaka Hospital, Takasaki, Gunma, Japan; ${ }^{2}$ Department of Internal Medicine, Mitsubishi Mihara Hospital, Mihara, Hiroshima, Japan; ${ }^{3}$ Junpukai Health Maintenance Center, Okayama, Okayama, Japan; ${ }^{4}$ Health Center, Shimane Environment and Health Public Corporation, Matsue, Shimane, Japan; ${ }^{5}$ Department of Internal Medicine, Meiwa Hospital, Nishinomiya, Hyogo, Japan; ${ }^{6}$ Department of Preventive Medicine, Matsue Red Cross Hospital, Matsue, Shimane, Japan; ${ }^{7}$ Public Health Center, Okazaki City Medical Association, Okazaki, Aichi, Japan; ${ }^{8}$ Department of Internal Medicine, Saiseikai Karatsu Hospital, Karatsu, Saga, Japan; ${ }^{9}$ Department of Gastroenterology and Hepatology, Shinko Hospital, Kobe, Hyogo, Japan; ${ }^{10}$ Department of Medical Innovation, Nagoya City University Graduate School of Medical Sciences, Nagoya, Aichi, Japan; ${ }^{11}$ Department of Laboratory Medicine, The Jikei University School of Medicine, Minato-ku, Tokyo, Japan; ${ }^{12}$ Department of Gastroenterology, Nippon Medical School Graduate School of Medicine, Bunkyo-ku, Tokyo, Japan; ${ }^{13}$ Department of General Internal Medicine 2, Kawasaki Medical School General Medical Center, Okayama, Japan; and ${ }^{14}$ Department of Gastroenterology and Metabolism, Nagoya City University Graduate School of Medical Sciences, Nagoya, Aichi, Japan
\end{abstract}

\section{Background/Aims}

Although risk factors of reflux esophagitis (RE) have been investigated in numerous cross-sectional studies, little is known about predictive factors associated with future onset of RE. We investigated time courses of clinical parameters before RE onset by a longitudinal case-control study using health checkup records.

\section{Methods}

We used health checkup records between April 2004 and March 2014 at 9 institutions in Japan. A multivariate logistic regression analysis was performed to evaluate associations of baseline clinical parameters with RE. The time courses of the clinical parameters of RE subjects were compared with those of non-RE subjects by the mixed-effects models for repeated measures analysis or longitudinal multivariate logistic analysis.

\section{Results}

Initial data were obtained from 230056 individuals, and 2066 RE subjects and 4132 non-RE subjects were finally included in the analysis. Body mass index, alanine aminotransferase, smoking, acid reflux symptoms, hiatal hernia, and absence of atrophic gastritis at baseline were independently associated with RE. The time courses of body mass index, fasting blood sugar, triglyceride, aspartate aminotransferase, alanine aminotransferase, $\gamma$-glutamyl transpeptidase, percentages of acid reflux symptoms, feeling of fullness, and hiatal hernia in the RE group were significantly worse than in the non-RE group.

\section{Conclusions}

The RE group displayed a greater worsening of the clinical parameters associated with lifestyle diseases, including obesity, diabetes, hyperlipidemia, and fatty liver for 5 years before RE onset compared with the non-RE group. These results suggest that RE is a lifestyle disease and thus lifestyle guidance to at-risk person may help to prevent RE onset.

(J Neurogastroenterol Motil 2022;28:86-94)

Key Words

Epidemiology; Esophagitis; Longitudinal studies; Risk factors 
Received: October 25, 2020 Revised: January 8, 2021 Accepted: January 19, 2021

(a) This is an Open Access article distributed under the terms of the Creative Commons Attribution Non-Commercial License (http://creativecommons. org/licenses/by-nc/4.0) which permits unrestricted non-commercial use, distribution, and reproduction in any medium, provided the original work is properly cited.

*Correspondence: Takeshi Kamiya, MD, PhD

Department of Medical Innovation, Nagoya City University Graduate School of Medical Sciences, 1 Kawasumi, Mizuho-cho, Mizuho-ku, Nagoya, Aichi 467-8601, Japan

Tel: +81-52-858-7215, Fax: +81-52-853-8321, E-mail: kamitake@med.nagoya-cu.ac.jp

\section{Introduction}

Reflux esophagitis (RE) is a disease in which the reflux of gastric contents into the esophagus causes superficial erosion of the mucosa of the lower esophagus, and its prevalence and burden have recently increased. ${ }^{1,2}$ Risk factors of $\mathrm{RE}$ proposed in previous studies included obesity, ${ }^{3-5}$ being male, ${ }^{4,6}$ hiatal hernia, ${ }^{7-9}$ absence of atrophic gastritis, ${ }^{10}$ advanced age, ${ }^{11}$ diabetes, ${ }^{12}$ drinking, ${ }^{4,9}$ smoking, ${ }^{4,912}$ metabolic syndrome, ${ }^{9,13,14}$ Helicobacter pylori negative, ${ }^{4,10}$ and high serum pepsinogen I/II ratio. ${ }^{4}$ However, these factors were found in cross-sectional studies and the systematic reviews based on them, and little is known about predictive factors associated with future RE onset because of a lack of longitudinal studies. Although 1 longitudinal study ${ }^{15}$ proposed that a $>1 \%$ increase in the body mass index (BMI) over 5 years, hiatal hernia, and drinking were risk factors of RE after 5 years, we cannot consider possible RE onset risk factors based on this report, because Azumi et $\mathrm{al}^{15}$ focused mainly on changes in the prevalence of reflux symptoms and RE over 5 years rather than the associated factors of future RE onset and thus did not exclude patients already with $\mathrm{RE}$ at the start of the follow-up. A further longitudinal study ${ }^{16}$ showed that being male, BMI $\geq 27 \mathrm{~kg} / \mathrm{m}^{2}$, smoking, drinking, hyperuricemia, and metabolic syndrome were risk factors of $\mathrm{RE}$ onset within 1 year. However, it remains unclear whether long-term changes of these factors are associated with future $\mathrm{RE}$ onset.

Patients with RE frequently suffer from troublesome symptoms, ${ }^{17}$ which also impair their health-related quality of life (HRQOL) ${ }^{18}$ While RE may also cause complications, including bleeding and stricture of the esophagus, ${ }^{19,20}$ and rarely esophageal adenocarcinoma following Barrett's esophagus, ${ }^{21}$ it is generally considered a chronic and non-life-threatening disease. Although it is difficult to conduct long-term cohort studies to focus on risk factors of future RE, retrospective studies can be implemented when consecutive health information for multiple years is available for clinical studies. Fortunately, in Japan, an annual health checkup is routinely conducted mainly for company employees, and upper gastrointestinal endoscopy is commonly performed even in healthy examinees.
Therefore, we conducted a multicenter retrospective study to investigate factors associated with future $\mathrm{RE}$ onset using long-term health checkup records in Japan.

\section{Materials and Methods}

This was a multicenter, retrospective, longitudinal study using health checkup records from 9 institutions in Japan. This study was conducted in accordance with the Declaration of Helsinki (7th revision, 2013), having been approved by the Central Ethics Committee of the Japanese Association for the Promotion of State-of-the-Art in Medicine, Nagoya, Aichi, Japan (Registration No. JAPSAM-2011). This study was registered with the University Hospital Medical Information Network Center Clinical Trials Registry in Japan (UMIN-CTR Registration No. UMIN000014640).

\section{Population}

Nine Japanese institutions, which met following criteria, participated in this study: (1) age, sex, body height, body weight, drinking, smoking, fasting blood sugar (FBS), hemoglobin A1c (HbAlc), and records of upper gastrointestinal endoscopy were available from health checkup records; and (2) health checkup records from at least 4 successive years were available. An initial dataset was obtained from individuals who participated in the health checkup and received at least 1 upper gastrointestinal endoscopy in 10 years (between April 2004 and March 2014) at the participating institutions. From individuals endoscopically diagnosed with RE, case candidates who met the following criteria were extracted: (1) participated in $\geq 4$ health checkups at the participating institutions between April 2004 and March 2014, (2) was newly-diagnosed with RE after April 2009, (3) had upper gastrointestinal endoscopy during the 2 years prior to the first RE diagnosis, (4) participated in $\geq 3$ health checkups in the 5 years before the first RE diagnosis, and (5) was $\geq 30$ years of age at the first RE diagnosis. From individuals without RE diagnosis, control candidates who met the following criteria were extracted: (1) participated in $\geq 4$ health checkups between April 2004 and March 2014 and (2) had upper 
gastrointestinal endoscopy between April 2009 and March 2014. In case-control matching, 2 control candidates who were matched for age, sex, and participating institutions with the corresponding case and underwent upper gastrointestinal endoscopy at the year when the corresponding case was endoscopically diagnosed as having RE were extracted.

\section{Measures}

For case subjects, data obtained at the first year of RE diagnosis, referred to as the baseline year, and 5 years before this year were used for the analysis. For control subjects, data obtained at the baseline year of the corresponding case and 5 years prior to this year were used for the analysis. The presence of RE was defined as having grade $\mathrm{A}$ or more severe erosion according to the Los Angeles classification $^{22}$ from endoscopic observation, or when the presence of $\mathrm{RE}$ was reported in the records if the Los Angeles grade was unavailable. To investigate factors associated with future RE onset, the following clinical parameters were extracted from the records: age, sex, BMI, FBS, HbA1c, abdominal circumference (AC), systolic blood pressure (SBP), diastolic blood pressure (DBP), triglyceride (TG), high-density lipoprotein cholesterol (HDL-C), low-density lipoprotein cholesterol (LDL-C), total cholesterol, uric acid (UA), aspartate aminotransferase (AST), alanine aminotransferase (ALT), $\gamma$-glutamyl transpeptidase ( $\gamma$-GTP), drinking, smoking, gastrointestinal symptoms (acid reflux symptoms, gastric pain, heavy stomach, feeling of fullness, belching, and abdominal pain), comorbidities (diabetes, hypertension, and hyperlipidemia), findings of upper gastrointestinal endoscopy (hiatal hernia, atrophic gastritis, and Barrett's esophagus), and H. pylori infection.

\section{Statistical Methods}

Baseline characteristics of the case group were compared with those of the control group using Student's $t$ test or chi-square test, as appropriate. A multivariate logistic regression analysis was performed to evaluate the effects of the baseline characteristics on presence or absence of RE. The independent variables for the multivariate logistic model were selected from the clinical parameters at baseline by stepwise forward-backward procedure, and multicollinearity of them were assessed using variance inflation factor. Missing values in the independent variables were complemented by multiple imputation method with chained equations. For each year before the baseline year, a cross-sectional comparison of the factors in both groups was made as for the baseline characteristics. To compare time-courses of the factors expressed as continuous variables

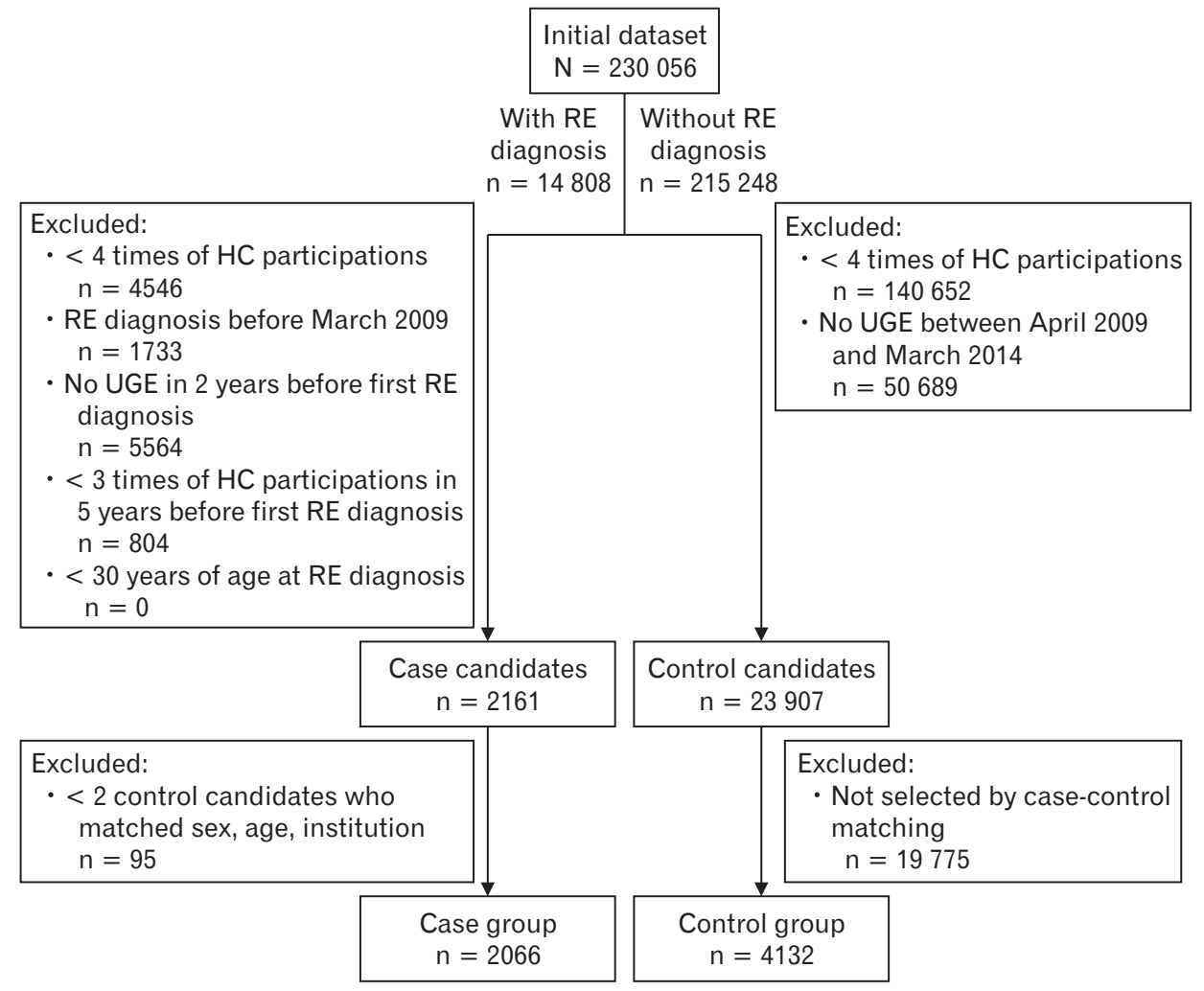

Figure 1. Subject flow in this study. $\mathrm{HC}$, health checkup; UGE, upper gastrointestinal endoscopy; RE, reflux esophagitis. 
between the case and control groups, the mixed-effect models for repeated measures with group, year, and the interaction effect of them as fixed effects and individual as a random effect were applied. In contrast, for the factors expressed as categorical variables, the multivariate logistic regression analysis with group, year, and the interaction effect of them as independent variables was applied. This analysis was also performed for dichotomized continuous variables between within and outside of normal ranges, where cut-off values of them were as follows: $\mathrm{BMI} \geq 25 \mathrm{~kg} / \mathrm{m}^{2}, \mathrm{AC} \geq 85 \mathrm{~cm}$ in males and $90 \mathrm{~cm}$ in females, FBS $\geq 110 \mathrm{mg} / \mathrm{dL}, \mathrm{HbA} 1 \mathrm{c} \geq 5.5 \%$, SBP $\geq 140 \mathrm{mmHg}$, DBP $\geq 90 \mathrm{mmHg}, \mathrm{TG} \geq 150 \mathrm{mg} / \mathrm{dL}$, HDL-C $<40 \mathrm{mg} / \mathrm{dL}, \mathrm{LDL}-\mathrm{C} \geq 120 \mathrm{mg} / \mathrm{dL}, \mathrm{UA}>7.0 \mathrm{mg} / \mathrm{dL}, \mathrm{AST}>$ $30 \mathrm{IU} / \mathrm{L}, \mathrm{ALT}>30 \mathrm{IU} / \mathrm{L}$, and $\gamma-\mathrm{GTP}>50 \mathrm{IU} / \mathrm{L}$. Additionally, analyses for subgroups aged $<55$ years and $\geq 55$ years at the baseline year were similarly performed as for the whole data. These statistical analyses were implemented using JMP 12.0.1 (SAS Institute, Cary, NC, USA) and R version 3.6.3 (R Foundation for Statistical Computing, Vienna, Austria) with mice package. Statistical significance was determined by 2 -sided manner and the significance level was set to 0.05 .

\section{Results}

\section{Baseline Characteristics}

In the case-control matching, 2066 cases and 4132 control subjects were finally included in the analysis (Fig. 1). Baseline characteristics in the case and control groups at the baseline year are presented in Tables 1 and 2. Age and sex, which were matching factors in this study, were similar between the groups. The following baseline characteristics were significantly different between the 2 groups: BMI, AC, FBS, SBP, TG, HDL-C, UA, AST, ALT, $\gamma$-GTP, drinking, smoking, percentage with symptoms (acid reflux symptoms and feeling of fullness), percentages of the presence of hiatal hernia and atrophic gastritis, and percentage of comorbidities (hypertension and hyperlipidemia). In the multivariate analysis for the baseline characteristics, higher BMI, higher ALT, smoking, presence of acid reflux symptoms, presence of hiatal hernia, and absence of atrophic gastritis were independently related to $\mathrm{RE}$ (Table 3). Variance inflation factors of all the independent variables were less than 1.2.

Table 1. Subject Characteristics of the Case and Control Groups at Baseline (Continuous Parameters)

\begin{tabular}{|c|c|c|c|c|c|}
\hline \multirow{2}{*}{ Characteristics } & \multicolumn{2}{|c|}{ Case group } & \multicolumn{2}{|c|}{ Control group } & \multirow{2}{*}{$P$-value ${ }^{\text {a }}$} \\
\hline & $\mathrm{N}$ & Mean $\pm \mathrm{SD}$ & $\mathrm{N}$ & Mean $\pm \mathrm{SD}$ & \\
\hline Age (yr) & 2066 & $53.9 \pm 9.3$ & 4132 & $54.0 \pm 9.4$ & 0.963 \\
\hline BMI (kg/m²) & 2063 & $23.5 \pm 3.3$ & 4126 & $22.9 \pm 3.0$ & $<0.001$ \\
\hline $\mathrm{AC}(\mathrm{cm})$ & 2026 & $84.2 \pm 9.1$ & 4050 & $82.5 \pm 8.6$ & $<0.001$ \\
\hline FBS (mg/dL) & 2052 & $102.7 \pm 18.1$ & 4103 & $101.4 \pm 16.9$ & 0.006 \\
\hline $\mathrm{HbA1c}(\%)$ & 1648 & $5.7 \pm 0.6$ & 3276 & $5.7 \pm 0.5$ & 0.321 \\
\hline $\mathrm{SBP}(\mathrm{mmHg})$ & 2026 & $112.4 \pm 22.3$ & 4052 & $110.9 \pm 22.0$ & 0.011 \\
\hline $\mathrm{DBP}(\mathrm{mmHg})$ & 2026 & $84.3 \pm 22.0$ & 4052 & $83.1 \pm 21.9$ & 0.052 \\
\hline $\mathrm{TG}(\mathrm{mg} / \mathrm{dL})$ & 2022 & $126.7 \pm 90.2$ & 4039 & $115.5 \pm 72.8$ & $<0.001$ \\
\hline HDL-C (mg/dL) & 2025 & $60.4 \pm 15.9$ & 4052 & $61.5 \pm 15.8$ & 0.007 \\
\hline LDL-C (mg/dL) & 1453 & $122.5 \pm 30.2$ & 2909 & $122.9 \pm 29.5$ & 0.669 \\
\hline $\mathrm{TC}(\mathrm{mg} / \mathrm{dL})$ & 1764 & $206.5 \pm 32.6$ & 3540 & $206.4 \pm 32.9$ & 0.889 \\
\hline $\mathrm{UA}(\mathrm{mg} / \mathrm{dL})$ & 1779 & $5.69 \pm 1.32$ & 3555 & $5.57 \pm 1.3$ & 0.002 \\
\hline AST (IU/L) & 2025 & $24.1 \pm 11.0$ & 4052 & $23.0 \pm 8.7$ & $<0.001$ \\
\hline ALT (IU/L) & 2025 & $26.2 \pm 18.2$ & 4052 & $23.5 \pm 14.7$ & $<0.001$ \\
\hline$\gamma-\mathrm{GTP}(\mathrm{IU} / \mathrm{L})$ & 2025 & $46.0 \pm 49.4$ & 4052 & $40.2 \pm 44.1$ & $<0.001$ \\
\hline
\end{tabular}

${ }^{a} P$-values were calculated using Student's $t$ test.

$\mathrm{N}$, total number of subjects in the group (except subjects whose characteristics were unknown); BMI, body mass index; AC, abdominal circumference; FBS, fasting blood sugar; HbA1c, hemoglobin A1c; SBP, systolic blood pressure; DBP, diastolic blood pressure; TG, triglyceride; HDL-C, high-density lipoprotein cholesterol; LDL-C, low-density lipoprotein cholesterol; TC, total cholesterol; UA, uric acid; AST, aspartate aminotransferase; ALT, alanine aminotransferase; $\gamma$-GTP, $\gamma$-glutamyl transpeptidase. 
Table 2. Subject Characteristics of the Case and Control Groups at Baseline (Categorical Parameters)

\begin{tabular}{|c|c|c|c|c|c|}
\hline \multirow{2}{*}{ Characteristics } & \multicolumn{2}{|c|}{ Case group } & \multicolumn{2}{|c|}{ Control group } & \multirow{2}{*}{$P$-value } \\
\hline & $\mathrm{N}$ & $\mathrm{n}(\%)$ & $\mathrm{N}$ & $\mathrm{n}(\%)$ & \\
\hline Male & 2066 & $1558(75.4)$ & 4132 & $3116(75.4)$ & 1.000 \\
\hline Current drinking & 1925 & $1379(71.6)$ & 3838 & $2680(69.8)$ & 0.156 \\
\hline Amount of alcohol & 1392 & & 2740 & & $<0.001$ \\
\hline$<20 \mathrm{~g} /$ day & & $652(46.8)$ & & $1418(51.8)$ & \\
\hline $20-40 \mathrm{~g} /$ day & & $442(31.8)$ & & $872(31.8)$ & \\
\hline $40-60 \mathrm{~g} /$ day & & $240(17.2)$ & & $344(12.6)$ & \\
\hline$\geq 60 \mathrm{~g} /$ day & & $58(4.2)$ & & $106(3.9)$ & \\
\hline Current smoking & 1923 & $471(24.5)$ & 3838 & $821(21.4)$ & 0.008 \\
\hline \multicolumn{6}{|l|}{ Symptoms } \\
\hline Acid reflux symptoms & 2057 & $239(11.6)$ & 4117 & $192(4.7)$ & $<0.001$ \\
\hline Gastric pain & 1627 & $90(5.5)$ & 3259 & $166(5.1)$ & 0.517 \\
\hline Heavy stomach & 1358 & $136(10.0)$ & 2715 & $234(8.6)$ & 0.144 \\
\hline Feeling of fullness & 1244 & $106(8.5)$ & 2487 & $167(6.7)$ & 0.046 \\
\hline Belching & 1137 & $75(6.6)$ & 2273 & $116(5.1)$ & 0.074 \\
\hline Abdominal pain & 488 & $24(4.9)$ & 976 & $46(4.7)$ & 0.863 \\
\hline \multicolumn{6}{|l|}{ Endoscopic findings } \\
\hline Hiatal hernia & 1960 & $458(23.4)$ & 3916 & $492(12.6)$ & $<0.001$ \\
\hline Atrophic gastritis & 1960 & $693(35.4)$ & 3917 & $1637(41.8)$ & $<0.001$ \\
\hline Barrett's esophagus & 1960 & $53(2.7)$ & 3916 & $127(3.2)$ & 0.258 \\
\hline \multicolumn{6}{|l|}{ Comorbidities } \\
\hline Diabetes & 2022 & $174(8.6)$ & 4040 & $294(7.3)$ & 0.068 \\
\hline Hypertension & 2022 & $417(20.6)$ & 4040 & $704(17.4)$ & 0.003 \\
\hline Hyperlipidemia & 2022 & $351(17.4)$ & 4040 & $620(15.4)$ & 0.044 \\
\hline H. pylori infection & 33 & $13(39.4)$ & 87 & $46(52.9)$ & 0.187 \\
\hline
\end{tabular}

${ }^{a} P$-values were calculated using chi-square test.

$\mathrm{N}$, total number of subjects in the group (except subjects whose characteristics were unknown); H. pylori, Helicobactor pylori.

Table 3. Multivariate Logistic Regression Analysis for Baseline Characteristics

\begin{tabular}{lcr}
\hline \multicolumn{1}{c}{ Characteristics } & Adjusted OR [95\% CI] & $P$-value \\
\hline BMI (per 1 kg/m ${ }^{2}$ increase) ${ }^{\mathrm{a}}$ & $1.05[1.03,1.07]$ & $<0.001$ \\
ALT (per 10 IU/L increase) $)^{\mathrm{a}}$ & $1.05[1.02,1.09]$ & 0.004 \\
Current smoking & $1.15[1.01,1.32]$ & 0.035 \\
Acid reflux symptoms & $2.72[2.22,3.33]$ & $<0.001$ \\
Hiatal hernia & $2.32[2.01,2.69]$ & $<0.001$ \\
Atrophic gastritis & $0.66[0.58,0.74]$ & $<0.001$ \\
\hline
\end{tabular}

${ }^{\mathrm{a}}$ Unit ORs are presented for continuous variables.

BMI, body mass index; ALT, alanine aminotransferase.

A higher OR indicates a higher risk of having reflux esophagitis given each characteristic.

\section{Longitudinal Comparisons}

The time courses of the following factors were significantly different between the 2 groups: BMI, AC, FBS, TG, HDL-C, AST, ALT, $\gamma$-GTP, and percentages with acid reflux symptoms, feeling of fullness, and hiatal hernia (Fig. 2). Results of longitudinal comparisons of the other (non-significant) factors are also presented in Figure 2. In the longitudinal comparisons of the dichotomized variables, no significant differences in the time courses were observed.

\section{Cross-sectional Comparisons}

The values of the factors in both the groups before the baseline year are shown in Supplementary Table 1. Regarding the factors that were significantly different in the comparison of baseline characteristics, most showed differences for $\geq 1$ year consecutively before the baseline: BMI, AC, TG, ALT, acid reflux symptoms, and atrophic gastritis for 5 years; AST for 4 years; and HDL-C, smoking, and hiatal hernia for 3 years; FBS, SBP, UA, $\gamma$-GTP, and hypertension for 1 year. Additionally, some of the dichotomized continuous parameters also showed consecutive differences before baseline: BMI, AC, and ALT for 5 years; TG for 4 years; and FBS and $\gamma$-GTP for 1 year. 
A Body mass index

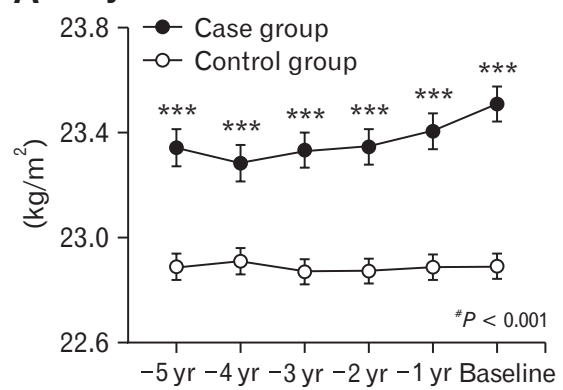

D Triglyceride

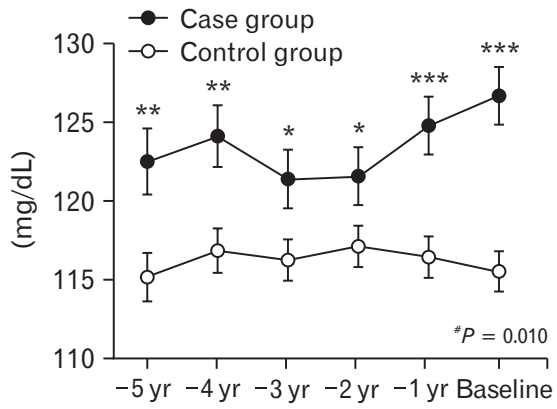

G ALT

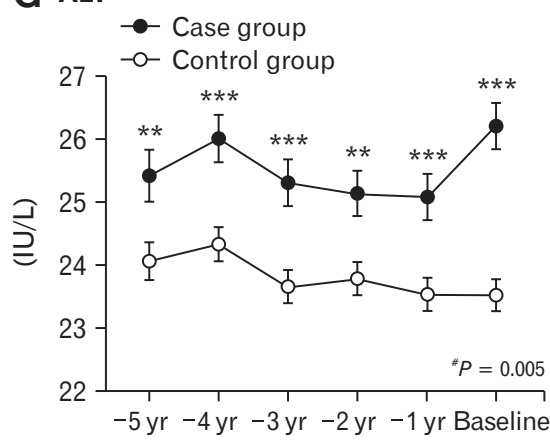

J Feeling of fullness

Case group

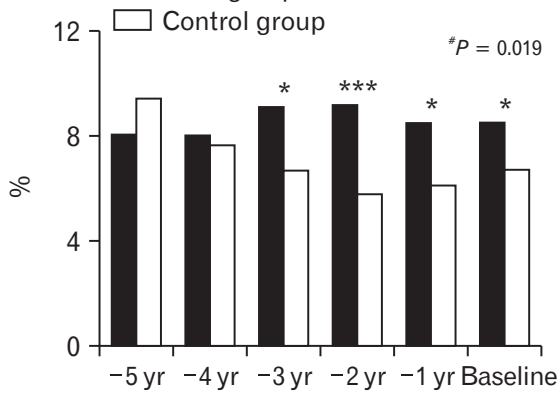

B Abdominal circumference

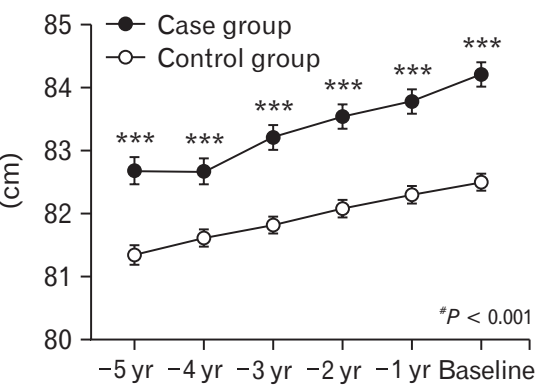

E HDL cholesterol

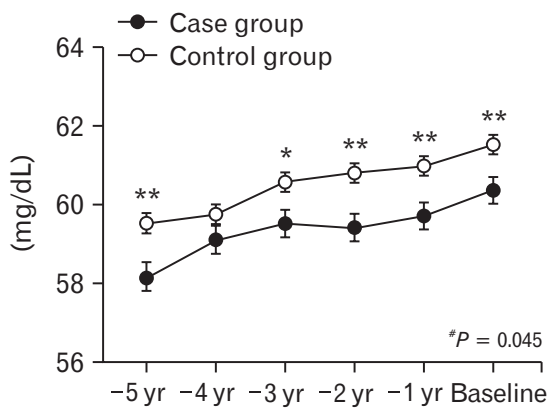

H $\gamma$-GTP

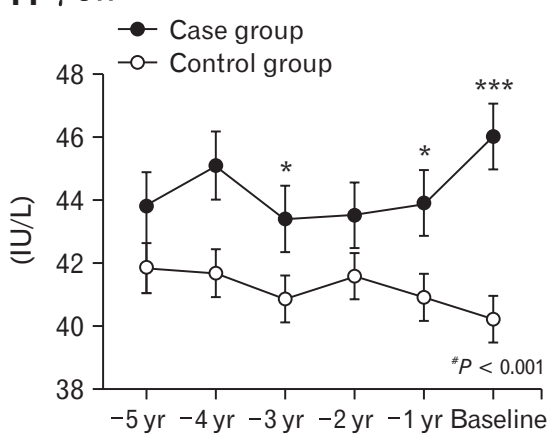

K Hiatal hernia

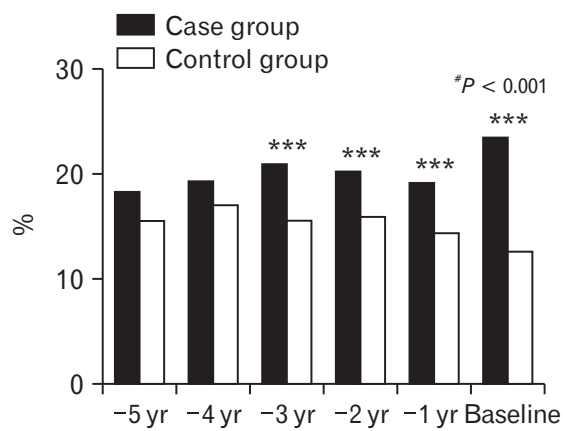

C Fasting blood sugar

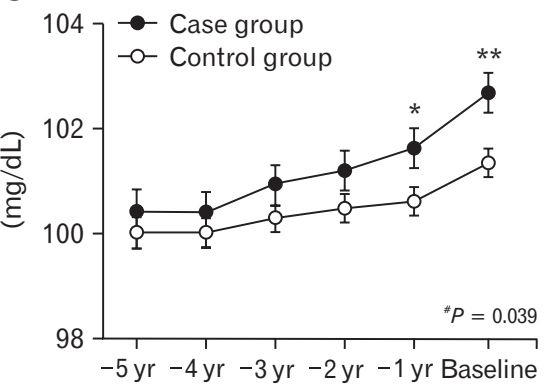

F AST

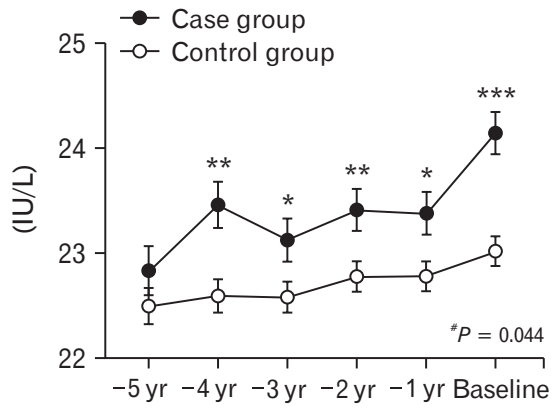

I Acid reflux symptoms

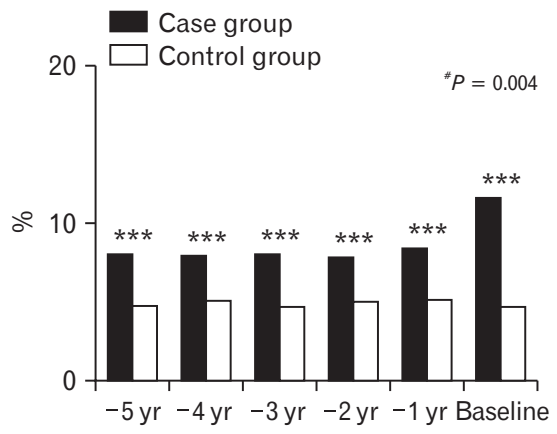

Figure 2. Time courses of the factors that were significantly different between the case and control groups in the longitudinal comparison. For continuous variables, points and error bars denote least square means at each year and its standard errors, respectively. For categorical variables, boxes denote percentages at each year. HDL, high-density lipoprotein; AST, aspartate aminotransferase; ALT, alanine aminotransferase; $\gamma$-GTP, $\gamma$-glutamyl transpeptidase. ${ }^{*} P<0.05 ;{ }^{*} P<0.01 ;{ }^{* *} P<0.001$ in the cross-sectional comparisons between the groups. ${ }^{*} P$-value in the longitudinal comparison between the groups. 


\section{Subgroup Analysis}

In the subgroup analysis for younger ( $<55$ years of age) subjects, the following baseline characteristics were significantly different between the groups: BMI, AC, FBS, SBP, DBP, TG, HDLC, UA, AST, ALT, $\gamma$-GTP, drinking, amount of alcohol consumed, smoking, acid reflux symptoms, stomach heaviness, hiatal hernia, atrophic gastritis, hypertension, and hyperlipidemia (Supplementary Table 2). Most of these differed for $\geq 1$ year consecutively before the baseline: BMI, AC, TG, ALT, $\gamma$-GTP, and acid reflux symptoms for 5 years; FBS, HDL-C, drinking, smoking, and hiatal hernia for 3 years; UA for 2 years; SBP and DBP for 1 year. The time courses of BMI, ALT, $\gamma$-GTP, and hiatal hernia were significantly different between the groups (Supplementary Table 3).

In the subgroup analysis for older ( $\geq 55$ years of age) subjects, the following baseline characteristics were significantly different between the groups: BMI, AC, TG, ALT, amount of alcohol consumed, acid reflux symptoms, hiatal hernia, atrophic gastritis, hypertension, and hyperlipidemia (Supplementary Table 4). Most of these differed for $\geq 1$ year consecutively before the baseline: BMI, AC, and atrophic gastritis for 5 years; acid reflux symptoms and hiatal hernia for 3 years; TG and ALT for 1 year. The time courses of BMI, AC, and TG were significantly different between the groups (Supplementary Table 5).

\section{Discussion}

This study was implemented using health checkup records for 10 years to investigate factors associated with future RE onset. The multivariate analysis for the baseline characteristics showed that higher BMI, higher ALT, smoking, presence of acid reflux symptoms, presence of hiatal hernia, and absence of atrophic gastritis were independent risk factors for RE, although the baseline characteristics mutually correlated and the unselected variables in this analysis may also be important risk factors. Significant differences in the time courses between the case and control groups were observed for BMI, the factors associated with metabolic syndrome, including AC, FBS, TG, and HDL-C, ${ }^{23-25}$ indices of hepatic function (AST, ALT, and $\gamma$-GTP), and the percentages with acid reflux symptoms, feeling of fullness, and hiatal hernia. In cross-sectional analysis, most of the factors displaying significant differences between the groups at the baseline year corresponded with the associated factors proposed by previous cross-sectional studies, with BMI, AC, TG, ALT, and the percentages with acid reflux symptoms and atrophic gastritis being constantly different throughout the 5 years before the baseline. Although the means of BMI, AC, TG, and ALT remained within the normal ranges even in the case group, the rates of subjects who had them outside of the normal ranges were significantly higher in the case group. The analysis for the younger subgroup displayed greater differences between the groups than in the older subgroup, except for the percentage with atrophic gastritis, although the results in both these groups were similar to those in the whole data.

In the longitudinal comparisons, factors showing significant differences in the time course between the groups included laboratory parameters related to lifestyle diseases. AC, FBS, TG, and HDL-C are considered criteria of metabolic syndrome while indices of hepatic function are applied as markers of hepatic damage caused mainly by excessive intake of fats, carbohydrates, and alcohol. Because these factors (except HDL-C, described below) increased in the case group more rapidly with time, this suggests a close association of worsening lifestyle with RE onset. A previous study ${ }^{16}$ also found high BMI, metabolic syndrome, and hyperuricemia to be significant risk factors of RE onset during the following year. Therefore, we suggest that RE is a lifestyle disease and lifestyle guidance to at-risk person could help prevent RE. Here, we should consider the contradictory result in that the HDL-C level, a higher value of which may counter lifestyle diseases, ${ }^{26}$ increased more rapidly in the case group. Although we cannot explain this result, we speculate that this was not a major result, because the $P$-value of 0.045 is only just significant, and the absolute HDL-C level in the case group at each year were consecutively lower than in the control group.

In the cross-sectional comparisons, most of factors displaying significant differences between the groups at baseline, including BMI, AC, FBS, SBP, TG, HDL-C, drinking, smoking, hiatal hernia, and atrophic gastritis, have previously been suggested to be associated with RE. ${ }^{8,11,16}$ We found that indices of hepatic function were also associated factors of RE, which have not been investigated in previous studies. Especially, it should be noted that higher ALT was identified as an independent risk factor also in the multivariate analysis for the baseline characteristics. Whereas our results of the cross-sectional comparison were similar to previous studies, we showed in the present study that BMI, AC, TG, ALT, and the percentages with acid reflux symptoms and atrophic gastritis had been significantly different between the groups for 5 years before the baseline year. Although we do not currently discuss how these factors are involved in the pathogenic process of $\mathrm{RE}$, these results suggest that further investigation of the long-term influences of risk factors on RE onset should be made. 
The younger subgroup clearly displayed greater differences, except for atrophic gastritis, between the groups in both longitudinal and cross-sectional comparisons compared with the older subgroup. This may result from the impact of lifestyle on RE onset being more direct in younger subjects, whereas it may become less clear in older subjects, for example, by a loss of the preventative mechanism against acid reflux caused through reasons other than lifestyle. Achem and DeVault ${ }^{27}$ reviewed that aging may predispose to gastroesophageal reflux disease and increase its severity via physiological changes, including weakened esophageal motility, reduced lower esophageal sphincter pressure, and poor primary/secondary peristalsis.

We show that the percentage with atrophic gastritis was significantly lower in the case group. Although the results of $H$. pylori test were almost unavailable in the health checkup records, we can assume a lower percentage of $H$. pylori-positive subjects in this group because atrophic gastritis is mainly caused by $H$. pylori infection. ${ }^{28}$ Therefore, our result is consistent with previous studies in which $H$. pylori infection and the presence of atrophic gastritis were reported to be negatively associated with RE. The declining $H$. pylori infection rate through expanded use of eradication therapy, particularly in developed countries, ${ }^{29}$ provides one possible contribution to the increasing number of RE patients. ${ }^{1}$ Considering the significant cost burden of RE and resulting poor HRQOL in patients, aggressive intervention in the lifestyle of those at risk of RE should be considered even before RE onset.

Because RE is not a life-threatening disease, no long-term cohort studies have been conducted to investigate risk factors of future RE. By using long-term health checkup records, we were able to investigate potential associated factors of future RE. The methodology of this study is appropriate to investigate risk factors of other chronic non-fatal diseases. However, non-standardized data formats used in different institutions may disturb studies using health checkup records. Indeed, our proposal of an association of lifestyle with RE onset was through "indirect" estimation based on the differences of the laboratory parameters between the groups, because lifestyle data could not be used in this study due to a lack of a common data format among the participating institutions. Should well-formatted lifestyle data be available, we could "directly" investigate the association. To initiate studies based on "real world data," including, for example, health checkup records and health insurance claims, standardization of the data format is essential.

The present study has limitations in addition to the non-uniformity of the data format. First, we defined the presence or absence of RE based solely on health checkup records between April 2004 and
March 2014, and were thus unable to determine whether subjects were diagnosed with RE before March 2004 or outside of health checkup. Therefore, non-newly diagnosed cases could be included in the case group, whereas subjects who had been diagnosed with $\mathrm{RE}$ before the examined records could be assigned to the control group. While this may have caused some bias, it was probably limited because the factors associated with RE in our study were consistent with those in previous studies. Secondly, we had planned to collect data regarding history of medications that could affect RE development, namely proton pump inhibitors, calcium blockers, and aspirin, at first; however we had to abandon this because such information were not adequately obtained by self-administered questionnaires in health checkups. Thus we could not have included the effect of medications in the analysis, and some biases probably exist in the assessment of presence or absence of RE, acid reflux symptoms, and the association between them. Finally, because we used health checkup records in this study, the subjects were considered to be relatively healthy compared with the general population of the same age. Therefore, our results cannot be generalized to a non-healthy population.

In conclusion, the factors thought to be related to lifestyle displayed significantly poorer values or larger worsening in $\mathrm{RE}$ patients than in non-RE subjects. Therefore, $\mathrm{RE}$ is thought to be a lifestyle disease, and lifestyle guidance should be given to health checkup participants with unhealthy lifestyle to prevent developing RE.

\section{Supplementary Materials}

Note: To access the supplementary tables mentioned in this article, visit the online version of Journal of Neurogastroenterology and Motility at http://www.jnmjournal.org/, and at https://doi. org/10.5056/jnm20237.

Acknowledgements: We are grateful to the GERD Society for administrative and clerical support of this study.

Financial support: This work was funded by the GERD Society Grant in 2014 supported by AstraZeneca K.K. (Osaka, Japan), based on a contract of clinical study support.

\section{Conflicts of interest: None.}

Author contributions: Koji Nakada, Katsuhiko Iwakiri, Ken Haruma, and Takashi Joh were involved in study conception and design; Yuzuru Toki, Ryo Yamauchi, Eizo Kayashima, Kyoichi Adachi, Kiyohiko Kishi, Hiroshi Suetsugu, Tsuneya Wada, Hi- 
royoshi Endo, and Hajime Yamada were involved in the acquisition of data; Satoshi Osaga, Takeshi Kamiya, Koji Nakada, Katsuhiko Iwakiri, Ken Haruma, and Takashi Joh were involved in the analysis and interpretation of data; and Satoshi Osaga and Takeshi Kamiya were involved in the drafting of the manuscript. All authors critically revised the draft and approved the final manuscript.

\section{References}

1. El-Serag HB, Sweet S, Winchester CC, Dent J. Update on the epidemiology of gastro-oesophageal reflux disease: a systematic review. Gut 2014;63:871-880.

2. Jung HK. Epidemiology of gastroesophageal reflux disease in Asia: a systematic review. J Neurogastroenterol Motil 2011;17:14-27.

3. Hampel H, Abraham NS, El-Serag HB. Meta-analysis: obesity and the risk for gastroesophageal reflux disease and its complications. Ann Intern Med 2005;143:199-211.

4. Minatsuki C, Yamamichi N, Shimamoto T, et al. Background factors of reflux esophagitis and non-erosive reflux disease: a cross-sectional study of 10,837 subjects in Japan. PLoS One 2013;8:e69891.

5. Adachi K, Mishiro T, Tanaka S, Hanada K, Kinoshita Y. Gender differences in time-course changes of reflux esophagitis in Japanese patients. Intern Med 2015;54:869-873.

6. Cook MB, Wild CP, Forman D. A systematic review and meta-analysis of the sex ratio for Barrett's esophagus, erosive reflux disease, and nonerosive reflux disease. Am J Epidemiol 2005;162:1050-1061.

7. Avidan B, Sonnenberg A, Schnell TG, Sontag SJ. Risk factors for erosive reflux esophagitis: a case-control study. Am J Gastroenterol 2001;96:4146.

8. Amano K, Adachi K, Katsube T, Watanabe M, Kinoshita Y. Role of hiatus hernia and gastric mucosal atrophy in the development of reflux esophagitis in the elderly. J Gastroenterol Hepatol 2001;16:132-126.

9. Gunji T, Sato H, Iijima K, et al. Risk factors for erosive esophagitis: a cross-sectional study of a large number of Japanese males. J Gastroenterol 2011;46:448-455.

10. Koike T, Ohara S, Sekine H, et al. Helicobacter pylori infection inhibits reflux esophagitis by inducing atrophic gastritis. Am J Gastroenterol 1999;94:3468-3472.

11. Lluch I, Ascaso JF, Mora F, et al. Gastroesophageal reflux in diabetes mellitus. Am J Gastroenterol 1999;94:919-924.

12. Labenz J, Jaspersen D, Kulig M, et al. Risk factors for erosive esophagitis: a multivariate analysis based on the ProGERD study initiative. Am J Gastroenterol 2004;99:1652-1656.

13. Chung SJ, Kim D, Park MJ, et al. Metabolic syndrome and visceral obesity as risk factors for reflux oesophagitis: a cross-sectional case-control study of 7078 Koreans undergoing health check-ups. Gut 2008;57:13601365 .

14. Niigaki M, Adachi K, Hirakawa K, Furuta K, Kinoshita Y. Associa- tion between metabolic syndrome and prevalence of gastroesophageal reflux disease in a health screening facility in Japan. J Gastroenterol 2013;48:463-472.

15. Azumi T, Adachi K, Arima N, et al. Five-year follow-up study of patients with reflux symptoms and reflux esophagitis in annual medical check-up field. Intern Med 2008;47:691-696.

16. Lee YC, Yen AM, Tai JJ, et al. The effect of metabolic risk factors on the natural course of gastro-oesophageal reflux disease. Gut 2009;58:174181.

17. Vakil N, van Zanten SV, Kahrilas P, Dent J, Jones R; Global consensus group. The Montreal definition and classification of gastroesophageal reflux disease: a global evidence-based consensus. Am J Gastroenterol 2006;101:1900-1920.

18. McDougall NI, Johnston BT, Kee F, Collins JS, McFarland RJ, Love AH. Natural history of reflux oesophagitis: a 10 year follow up of its effect on patient symptomatology and quality of life. Gut 1996;38:481-486.

19. Wienbeck M, Barnert J. Epidemiology of reflux disease and reflux esophagitis. Scand J Gastroenterol Suppl 1989;156:7-13.

20. Sakaguchi M, Manabe N, Ueki N, et al. Factors associated with complicated erosive esophagitis: a Japanese multicenter, prospective, crosssectional study. World J Gastroenterol 2017;23:318-327.

21. Haggitt RC, Tryzelaar J, Ellis FH, Colcher H. Adenocarcinoma complicating columnar epithelium-lined (Barrett's) esophagus. Am J Clin Pathol 1978;70:1-5.

22. Lundell LR, Dent J, Bennett JR, et al. Endoscopic assessment of oesophagitis: clinical and functional correlates and further validation of the Los Angeles classification. Gut 1999;45:172-180.

23. National cholesterol education program (NCEP) expert panel on detection, evaluation, and treatment of high blood cholesterol in adults (adult treatment panel III). Third report of the national cholesterol education program (NCEP) expert panel on detection, evaluation, and treatment of high blood cholesterol in adults (adult treatment panel III) final report. Circulation 2002;106:3143-3421.

24. Alberti KG, Zimmet P, Shaw J; IDF Epidemiology task force consensus group. The metabolic syndrome--a new worldwide definition. Lancet 2005;366:1059-1062.

25. Matsuzawa Y. Metabolic syndrome--definition and diagnostic criteria in Japan. J Atheroscler Thromb 2005;12:301.

26. Wannamethee SG, Shaper AG, Ebrahim S. HDL-cholesterol, total cholesterol, and the risk of stroke in middle-aged British men. Stroke 2000;31:1882-1888.

27. Achem SR, DeVault KR. Gastroesophageal reflux disease and the elderly. Gastroenterol Clin North Am 2014;43:147-160.

28. Kuipers EJ, Klinkenberg-Knol EC, Vandenbroucke-Grauls CM, Appelmelk BJ, Schenk BE, Meuwissen SG. Role of Helicobacter pylori in the pathogenesis of atrophic gastritis. Scand J Gastroenterol Suppl 1997;223:28-34.

29. Bruce MG, Maaroos HI. Epidemiology of Helicobacter pylori infection. Helicobacter 2008;13(suppl 1):1-6. 